\title{
CHEMICAL HISTORY WITH A NUCLEAR MICROPROBE
}

\author{
C.J. Maggiore, T.M. Benjamin, P.J. Hyde, \\ P.S.Z. Rogers, S. Srinivasan, J. Tesmer \\ Los Alamos National Laboratory, Los Alamos NM
}

87545

D.S. Woolum, D.S. Burnett

California Institute of Technology, Pasadena, CA

91125

$\underline{\text { Summary }}$

A nuclear microprobe cannot give direct information on the chemical state of an element, but the spatial distribution of elements in a specimen is often determined by the chemical history of the sample. Fuel cells and minerals are examples of complex systems whose elemental distributions are determined by past chemical history. The distribution of catalyst in used fuel cell electrodes provides direct information on the chemical stability of dispersed catalysts under operating conditions. We have used spatially resolved Rutherford backscattering to measure the migration of platinum and vanadium from intermetallic catalysts and to determine their suitability for use under the extreme operating conditions found in phosphoric acid fuel cells. Geologic materials are complex, heterogeneous samples with small mineral grains. The trace element distribution within the individual mineral grains and between different mineral phases is sensitive to the details of the mineral formation and history. The spatial resolution and sub-100-ppm sensitivity available with a nuclear microprobe open up several new classes of experiments to the geochemist. Geochemistry and electrochemistry are two areas proving particularly fruitful for application of the nuclear microprobe.

\section{Introduction}

The we11-known advantages of a nuclear microprobe are the sensitivity of particle-induced $x$-ray emission (PIXE) relative to an electron probe, the depth information available with Rutherford backscattering (RBS), light isotope detection with nuclear reactions, and the relatively unambiguous interpretation of the data. The two-and three-dimensional spatial resolution combined with trace element and monolayer sensitivity enables us to investigate a broad class of problems where the elemental distribution is modified by the chemical environment. The chemisorption of species from the liquid or gas phase onto solid surfaces, the stability of materials in extreme or changing chemical environments, and the kinetics of crystallization are problems where the past chemical history determines the present elemental distribution. A large number of related problems that cannot be solved simply without the nuclear microprobe exist in electrochemistry and geochemistry.

\section{Electrochemistry}

Two classes of electrochemical problems are amenable to investigation with a nuclear microprobe: stability of materials and composition of the solidliquid interface. Electrochemical systems of technological interest such as batteries or fuel cells are complex arrangements of electrodes, electrolytes, catalysts, and support structures operating in extreme chemical environments, where small changes in the composition or distribution of components can have a large effect on the efficiency or life of the system. The Los Alamos nuclear microprobe has been used to investigate the distribution and stability of platinum and platinum vanadium catalysts in new and used fuel cell electrodes.
The electrodes in a phosphoric acid fuel cell consist of a thin layer ( $120 \mu \mathrm{m})$ of platinum catalyzed carbon which is Teflon bonded and supported on a porous carbon backing. Cost considerations dictate that the noble metal loading be minimized and the migration, sintering, and corrosion of the catalyst be controlled. The present platinum level of $0.5 \mathrm{mg} / \mathrm{cm}^{2}$ or less means that the platinum is marginally detectable if at all with an electron microprobe. Figure 1 shows the $x$-ray spectrum obtained with a $5 \mu \mathrm{m}$-diam beam of 2.5-MeV protons incident on such an electrode. By scanning the surface of the electrode in $50 \mu \mathrm{m}$ steps, the uniformity of the platinum distribution was readily determined (Fig. 2 ).

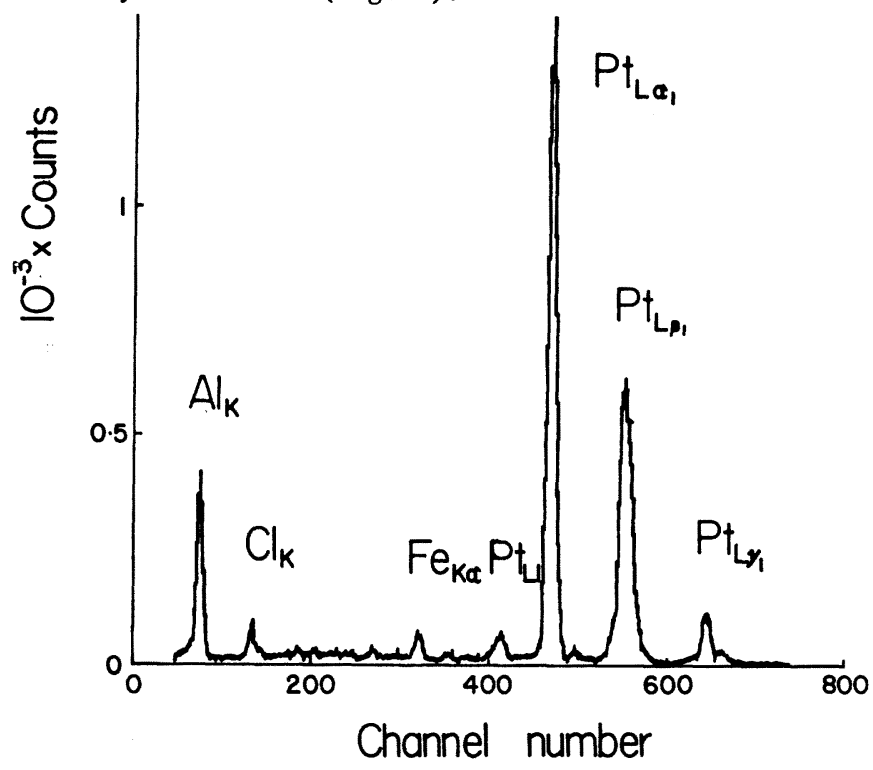

Figure 1. X-ray spectrum obtained from a $5 \mu \mathrm{m}$ beam of $2.5-\mathrm{MeV}$ protons on a carbon electrode with 0.5 $\mathrm{mg} / \mathrm{cm}^{2}$ platinum catalyst.

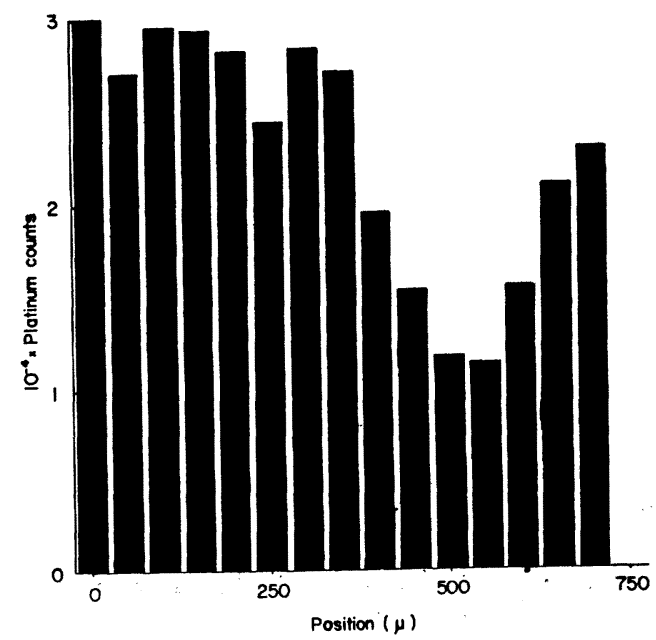

Figure 2. Platinum distribution as a function of position on the electrode determined by PIXE. 
This particular electrode was prepared by a postcatalyzation process using chloroplatinic acid followed by heat treatment in air. Questions of the uniformity of this method of catalyzing an electrode have always existed and with good reason as the results indicate. By simultaneously detecting the backscattered protons, the distribution of platinum below the surface of the electrode was also determined. Figure 3 shows the RBS spectrum obtained at a single point. The platinum concentration in the top $20 \mu \mathrm{m}$ of the electrode could be measured in this way. Figures 4 and 5 show isometric views of the data, indicating significant problems in the efficient use of the platinum catalyst.

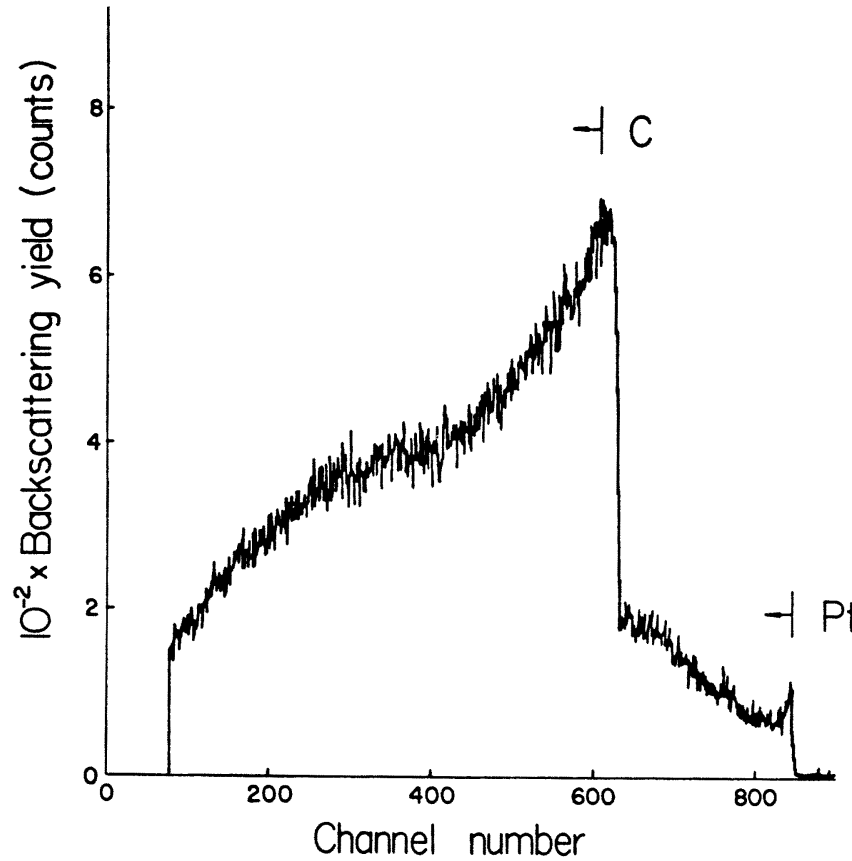

Figure 3. Rutherford backscattering spectrum from a post catalyzed electrode. The data were obtained with a $5 \mu \mathrm{m}$ beam of $2.5-\mathrm{MeV}$ protons.

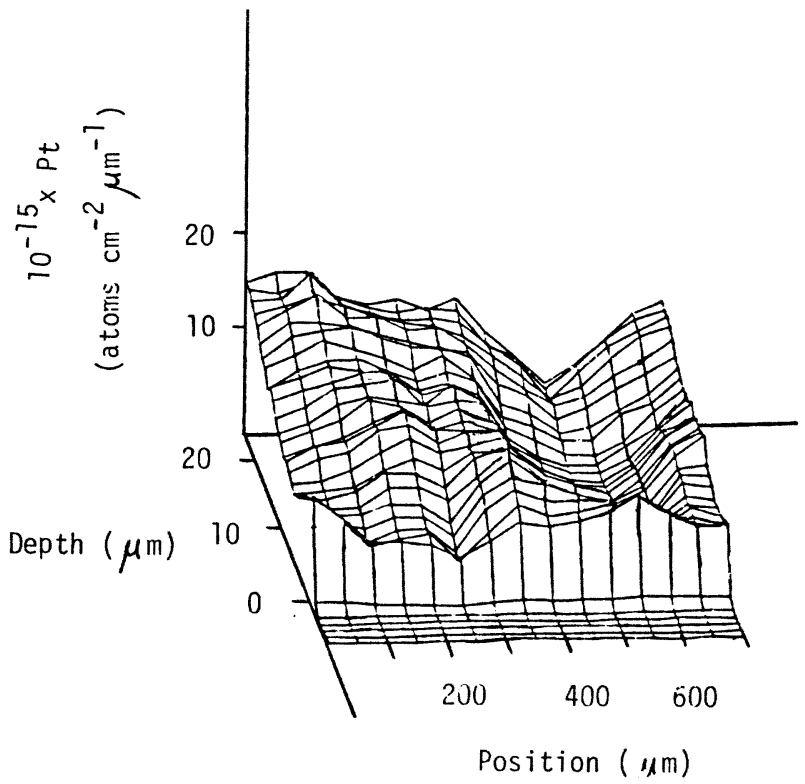

Figure 4. Isometric view of the platinum concentration vs. depth and position for a fuel cell electrode.

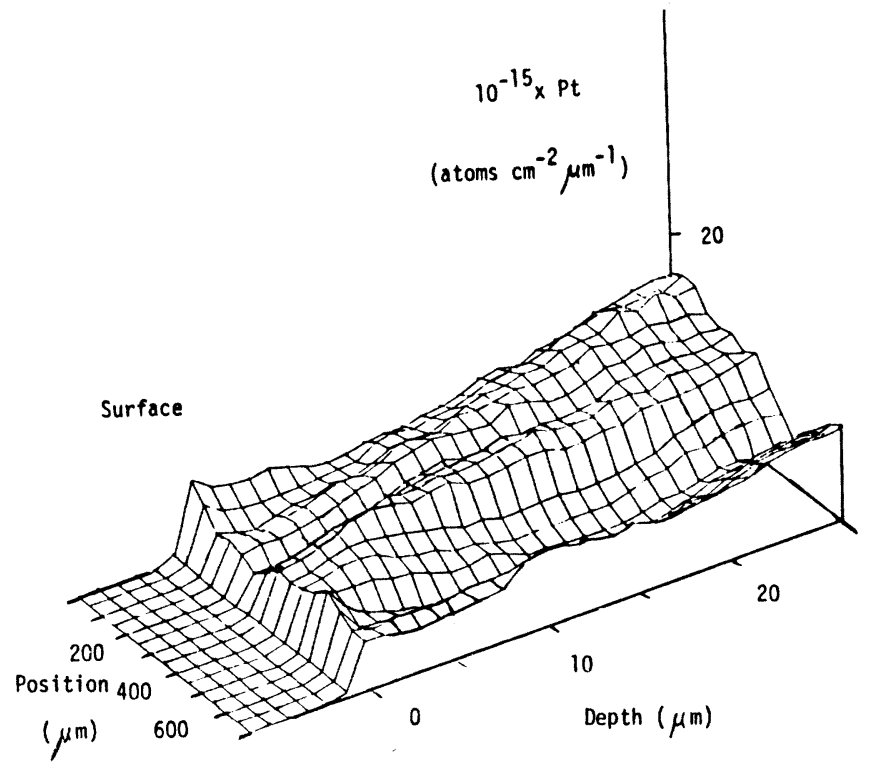

Figure 5. The same data as Fig. 4, but shown from a different viewing angle.

The efficiency of phosphoric acid fuel cells is limited by the slowness of the oxygen reduction reaction occurring at the cathode. Attempts have been made to improve cathode activity by using intermetallic platinum-vanadium as the catalyst. The intermetallic compound shows an initial activity for oxygen reduction greater than that of platinum, but during operation activity decreases to that of pure metal. The reason for this decreased activity was not understood until we studied the distribution of catalysts in the anode and cathode in new and used electrodes.

Figure 6 outlines the experiment. Small-angle cuts through the complete electrode structures were made, and line scans across the levels were performed. With the spatially resolved RBS, we were able to measure both the platinum and vanadium distributions throughout the electrodes. The results are shown in Figure 7. The vanadium component of the platinumvanadium catalyst is not stable. At the operating potential of the fuel cell, the vanadium dissolves in the phosphoric acid, migrates through the silicon carbide matrix, and accumulates near the anode. The nuclear microprobe was able to determine the chemistry of what happened in the fuel cell by measuring the elemental distribution in the used electrode.

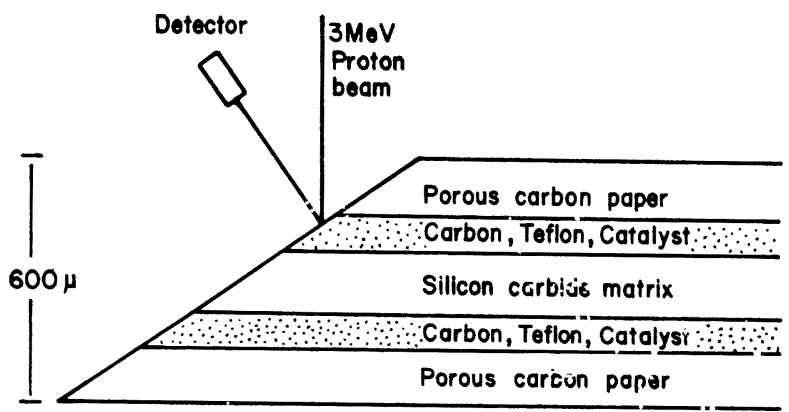

Figure 6. Experimental setup for using the nuclear microprobe to determine the distribution of catalyst in fuel cell electrodes. 


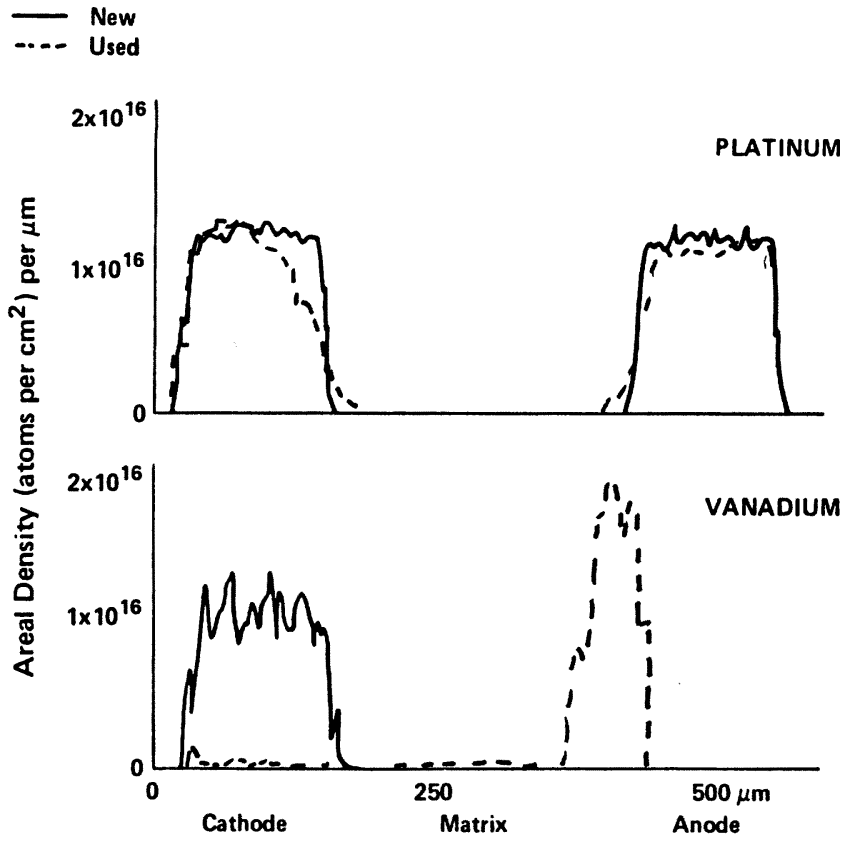

Figure 7. The distribution of $\mathrm{Pt}$ and $\mathrm{V}$ in new and used fuel cell electrodes.

One of the major problems in electrochemistry is the difficulty in obtaining direct compositional information at the solid-1iquid interface under realistic operating conditions. The $\mathrm{MeV}$ beams offer the possibility of probing this interfacial region through the solid if it can be made thin enough. Energy straggling through a foil less than a micrometer thick limits the depth resolution to much greater than a monolayer, but if the concentration at the interface is greater than the concentration in the liquid, the backscattering or nuclear reaction peak from the adsorbate can be assumed to come from the solid-liquid interface. Figure 8 shows the proposed experiment schematically. The electrode must be thin enough to allow measurement from the back side. Because the electrode must support atmospheric pressure, the aperture defining the free area of the foil electrode is small. Simple theory and experience with supported windows suggests that sub-micrometer-thick windows of a few hundred square micrometer can be made pinholefree. Our initial experiments will involve absolute coverage measurements of underpotentially deposited metals on electrodes.

\section{Geochemistry}

Geologic materials are complex, heterogeneous mixtures of minerals, of ten small grained and each with a different composition. Study of these materials demands an instrument capable of providing spatially resolved, in situ elemental analyses. One such instrument, the electron microprobe can analyze individual mineral phases in polished sections. It is entirely adequate to the task if a sensitivity of $1000 \mathrm{ppm}$ is sufficient, that is, for major element chemistry. However, in situ trace element ( $<1000 \mathrm{ppm})$ microanalysis provides a new dimension to petrology and geochemistry.

In situ analysis, as opposed to physical and chemical separations, is important so that trace element data can be interpreted in a petrographic context. The ambiguities associated with the purity of mineral separates can be avoided, comparisons of inter- and intra-grain variations can be made, and the importance of inclusions can be assessed. The

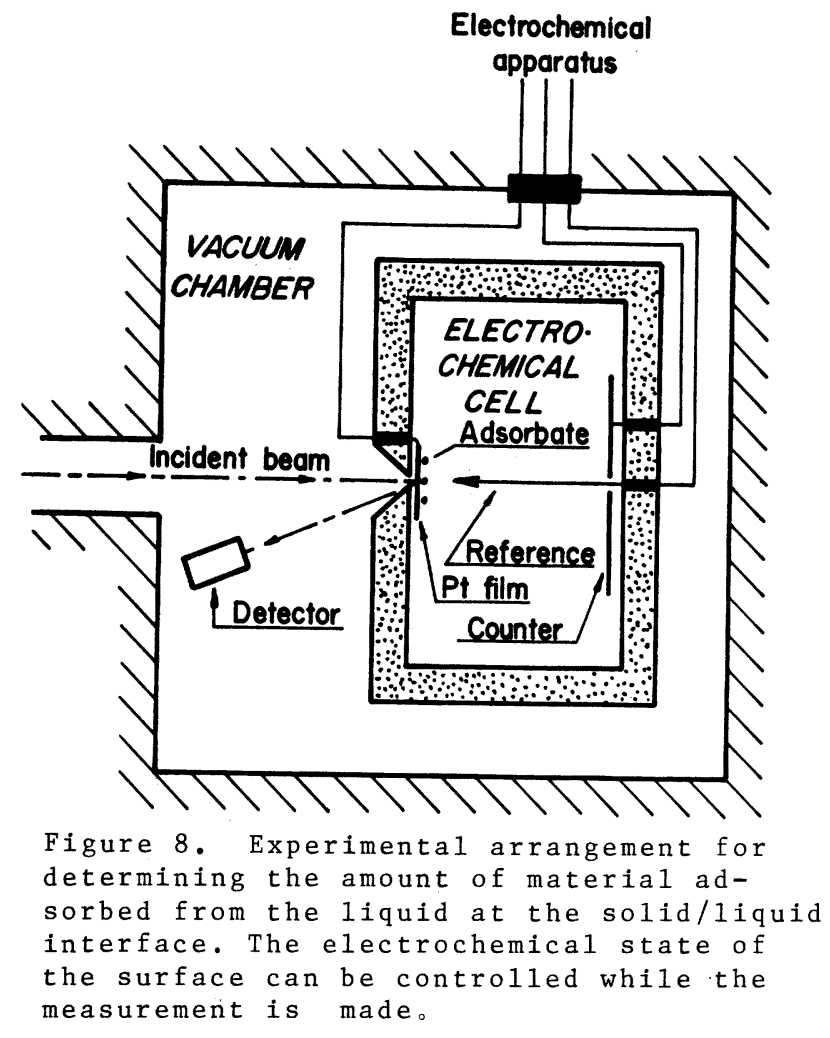

trace element sensitivity enables one to obtain detailed information on how the minerals were formed. By definition, trace elements either do not easily fit in the crystallographic lattice or were not present in large amounts in the magma when the mineral was formed. Their presence or absence in a particular mineral grain depends on the heating and cooling rates during formation and the diffusion, dissolution, ion exchange, and leaching after formation. In other words, the history of a mineral, its formation and subsequent environment, is contained in its trace element distribution.

We have used the Los Alamos nuclear microprobe for PIXE experiments on four categories of materials: geologic standards, terrestrial, meteoritic, and synthetic. We have addressed a broad range of problems to help define the limits of microprobe experiments for geochemistry. The meteoritic examples are the most general and will be discussed in somewhat more detail than the others.

The initial Pu/U content of the solar system is an unresolved problem in cosmochemistry. The production of plutonium and uranium occurs at a fairly constant ratio under a wide range of conditions in supernovae. The difference between the production ratio and the initial solar system ratio measures the time scale between $r$-process nucleosynthesis and solar system condensation by means of the $8.2 \times 10^{7}$ year half-life of $244 \mathrm{Pu}$. Another application of $\mathrm{Pu} / \mathrm{U}$ is as a relative chronometer of meteorite and lunar samples. The higher ratios come from older samples, and the age difference resolution by this method is potentially much better than that obtained by using absolute age chronometers such as $\mathrm{U} / \mathrm{Pb}$ and $\mathrm{Rb} / \mathrm{Sr}$.

The problem is that ${ }^{244} \mathrm{Pu}$ requires normalizing the plutonium to another element still present in the meteorite. Thus the problem becomes one of geochemical fractionation of different elements during geologic processes. The most tractable approach is to determine which meteorites, or more specifically, which phases within a meteorite, have unfractionated $\mathrm{Pu} / \mathrm{U}$ values. I 
Plutonium is anticipated to have the same trivalent charge as the light rare earth elements (LREE) and an ionic size between cerium and praseodymium. It should behave like the LREE, and experimental partition coefficients indicate that plutonium partitions to an extent intermediate between uranium and the LREE. A proposed "bracketing theorem" states that if uranium and LREE are not fractionated relative to each other in a particular meteoritic mineral, then the plutonium was not fractionated relative to the uranium in that mineral grain. Such a grain would be appropriate for relative $\mathrm{Pu} / \mathrm{U}$ dating. The nuclear microprobe is able to measure the LREE concentrations in individual mineral grains. Bulk analyses are not adequate; the particular mineral grain within the meteorite must be selected and proved to be unfractionated.

The enstatite chondrite Pena Blanca Spring (E6) contains the mineral oldhamite, a $\mathrm{CaS}$ phase that is the primary host of thorium and uranium in this meteorite. The $T h / U$ values are cosmic, indicating a lack of Th-U fractionation; and excess fossil fission tracks in the oldhamite have been attributed to $244 \mathrm{Pu}$. It if could be demonstrated that U-LREE fractionation did not occur, then the enstatite chondrite would be a good candidate for determining the initial $\mathrm{Pu} / \mathrm{U}$ value in the solar system. We used the nuclear microprobe to determine the LREE abundances in the oldhamite, $230 \mathrm{ppm}$ cerium and 220 ppm neodymium. Unfortunately, the LREE enrichment factor for this oldhamite grain is 245 while the uranium enrichment factor is 2200 . Thus, U-LREE fractionation did occur, and the Pena Blanca Spring meteorite is not a good candidate for $\mathrm{Pu} / \mathrm{U}$ dating. Another chondrite, St. Severin (LL6), contains two phosphate phases, whitlockite and chlorapatite. Our nuclear microprobe analyses of these phases yielded LREE concentrations of $1100 \mathrm{ppm}$ in the whitlockite and concentrations below our limits of detectability in chlorapatite. Bulk analyses were not able to determine unambiguously whether fractionation occurred. Because plutonium is geochemically associated with LREE, this in situ trace element microanalysis indicates that whitlockite is the appropriate phase for plutonium chronometry studies.

The limitations of micro-PIXE analysis on geologic materials are set by the constraints of specimen preparation and $x$-ray spectrometer resolution. Routine thin sections for a geologist are $30 \mu \mathrm{m}$ thick, and it is difficult to work with unsupported sections or sections thinner than this. The sensitivity limits observed with biological thin sections are probably an order of magnitude lower than routinely available with realistic geologic samples. As Fig. 9 shows, the $\mathrm{Si}(\mathrm{Li})$ detector energy resolution is not adequate to completely resolve the numerous L lines of the LREE. A focusing crystal spectrometer has the necessary energy resolution, but the reduced efficiency and single-element analysis mean slower data acquisition. This can partially be overcome by the higher current density available with the Los Alamos microprobe. In fact, all data taken with a $S i(L i)$ detector have been with significantly reduced beam currents to avoid pile-up problems. The scattering chamber is being redesigned to accommodate two focusing crystal spectrometers.

The microprobe has been used to study a broad range of other geochemical problems including trace element mobilization as a result of in situ retorting of oil shale, trace element fractionation anomalies in a suite of volcanic rocks, geochemistry of waterrock interactions, and partitioning coefficients in synthetic silicate systems. In all cases, the nuclear microprobe has enabled the geochemist to obtain in-

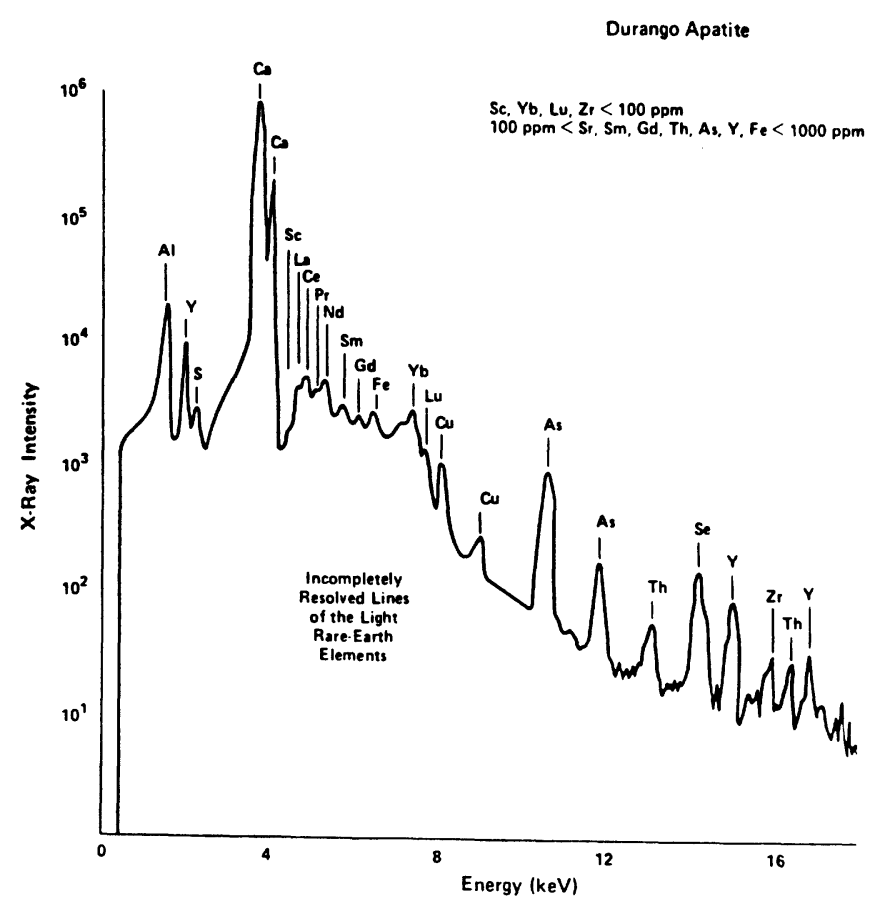

Figure 9 Results of nuclear microprobe analysis of Durango apatite, a calcium fluorophosphate containing a large number of rare earths. X-ray emission was induced by a beam of $3-\mathrm{MeV}$ protons.

formation on the behavior of trace elements within individual mineral grains still in their original petrographic context.

\section{Acknowledgments}

The authors wish to acknowledge the help and support of the Laboratory's Van de Graaff group. This work was supported by the United States Department of Energy.

\section{$\underline{\text { References }}$}

${ }^{1}$ D.S. Burnett and D.S. Woolum, In Situ Trace Element Microanalysis, Annual Reviews of Earth and Planetary Science, 1982. 